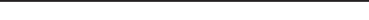

口-

Fuzzy set theory is an effective alternative to probability theory in solving many problems of studying processes and systems under conditions of uncertainty. The application of this theory is especially in demand in situations where the system under study operates under conditions of rapidly changing influencing parameters or characteristics of the environment. In these cases, the use of solutions obtained by standard methods of the probability theory is not quite correct. At the same time, the conceptual, methodological and hardware base of the alternative fuzzy set theory is not sufficiently developed. The paper attempts to fill existing gaps in the fuzzy set theory in some important areas. For continuous fuzzy quantities, the concept of distribution density of these quantities is introduced. Using this concept, a method for calculating the main numerical characteristics of fuzzy quantities, as well as a technology for calculating membership functions for fuzzy values of functions from these fuzzy quantities and their moments is proposed. The introduction of these formalisms significantly extends the capabilities of the fuzzy set theory for solving many real problems of computational mathematics. Using these formalisms, a large number of practical problems can be solved: fuzzy regression and clustering, fuzzy multivariate discriminant analysis, differentiation and integration of functions of fuzzy arguments, state diagnostics in a situation where the initial data are fuzzy, methods for solving problems of unconditional and conditional optimization, etc. The proof of the central limit theorem for the sum of a large number of fuzzy quantities is obtained. This proof is based on the characteristic functions of fuzzy quantities introduced in the work and described at the formal level. The concepts of independence and dependence for fuzzy quantities are introduced. The method for calculating the correlation coefficient for fuzzy numbers is proposed. Examples of problem solving are considered

Keywords: distribution density of fuzzy quantities, moments, characteristic functions, limit theorem, correlation coefficient
口-
UDC 519.85

DOI: $10.15587 / 1729-4061.2020 .217630$

\section{DEVELOPMENT OF METHODS FOR EXTENSION OF THE CONCEPTUAL AND ANALYTICAL FRAMEWORK OF THE FUZZY SET THEORY}

\author{
L. Raskin \\ Doctor of Technical Sciences, Professor* \\ E-mail: topology@ukr.net \\ 0. Sira \\ Doctor of Technical Sciences, Professor* \\ E-mail: topology@ukr.net \\ *Department of Distributed Information \\ Systems and Cloud Technologies \\ National Technical University \\ «Kharkiv Polytechnic Institute» \\ Kyrpychova str., 2, Kharkiv, Ukraine, 61002
}

Copyright (C) 2020, L. Raskin, O. Sira

This is an open access article under the CC BY license (http://creativecommons.org/licenses/by/4.0)

\section{Introduction}

The recent increase in the number of publications on solving problems in conditions of uncertainty is objectively motivated. One of the important reasons for this is an increasingly clear understanding of insufficient validity of the routine use of traditional methods and technologies of the probability theory in solving practical problems under conditions of uncertainty in economics, technology, sociology, etc. A characteristic feature of the problems of studying phenomena and objects of the real world is the heterogeneity of the initial data. Due to the variability of the most important defining properties and characteristics of the environment, practical problems are formulated and solved in conditions of small samples of initial data, when fundamental provisions, axiomatic foundations of the probability theory are violated. A change in the mechanism of formation of the randomness phenomenon leads to heterogeneity of samples containing a small number of elements that are not sufficiently representative of the general population. The fuzzy set theory developed in [1] is much less demanding on the quality of the initial data, allows solving practical problems and drawing fairly reasonable conclusions for real samples.
Uncertainty is an inherent quality of the real world. Modern models, methods and technologies for solving practical problems are based on the implementation of two conceptually different approaches to describing this uncertainty: probability theory and fuzzy set theory. Thus, the fuzzy set theory (FST) [1-5] is a successful and productive alternative to the probability theory (PT) in situations where the mechanism of formation of random variables changes unpredictably. This precludes the use of PT methods for the analytical construction of appropriate distribution densities or reconstructing them from experimental data in these cases.

At the same time, PT has significant advantages over FST, which are determined by the presence of deep and constructive probabilistic formalisms [2]: moment theory arising from the existence of distribution densities of random variables (RV); the possibility to calculate the probabilities of falling of RV in given subsets of a set of possible values; calculation of numerical characteristics of functions random variable functions; use of characteristic functions of random variables; the existence of the central limit theorem, etc. This circumstance stimulates attempts to formally extend the axiomatic base of FST in order 
to replenish the mathematical framework of this theory for solving practical problems.

\section{Literature review and problem statement}

There are a very large number of papers on the fuzzy set theory. These works can be divided into two groups. The first one includes works containing a presentation of the fundamental foundations of this modern mathematical framework [1-8]. In the works of the second group, the application of this theory is considered [9-13]. In recent years, there has been an increase in the number of papers that consider examples of solving various practical problems. At the same time, the number of problems solved is impressive. However, many of the most serious works [1-4], as well as subsequent works, draw attention to the need to improve the axiomatic base of the fuzzy set theory. For example, the papers $[5,14-16]$ on the analysis of the mathematical foundations of this theory explicitly state the need to continue efforts to improve the fundamental principles and applied analytical methods of the fuzzy set theory. Other considerations encouraging work in this direction draw attention to the following [17-19]. Implementation of solutions obtained by traditional methods of the probability theory in situations where the basic principles governing the possibility of applying this theory are violated in the collection of statistical material is incorrect, and in many cases even dangerous.

It is clear that throughout the past time, the framework of the fuzzy set theory has been continuously replenished. At the same time, the main efforts were aimed at developing the most often used sections of the theory. These are operations on fuzzy sets and fuzzy numbers, fuzzy relations, fuzzy logic, fuzzy inference systems, simple approaches to solving fuzzy optimization problems. However, some important issues of the fuzzy measure theory remain insufficiently studied. In this regard, using canonized approaches implemented in the probability theory, it is advisable to formulate the problem of developing a system of conceptual, axiomatic and framework formalisms in order to improve the methodological and model base, as well as the analytical framework of the fuzzy set theory. This concerns, in particular, the following issues: calculation of a fuzzy analogue of the distribution density of fuzzy numbers and their moments, consideration of the concepts of independence and dependence for fuzzy numbers, methods for calculating characteristic functions of fuzzy quantities, study of the limit characteristics of the sum of a large number of independent fuzzy quantities.

\section{The aim and objectives of the study}

The aim of the study is to extend the conceptual and analytical framework of the fuzzy set theory.

To achieve the aim, the following objectives are proposed:

- to develop a scheme for calculating the distribution density of a continuous fuzzy quantity, the expected value of the fuzzy quantity, the expected value of the fuzzy quantity function;

- to substantiate the concepts of independence and dependence for fuzzy quantities, derive a formula for calculating the correlation coefficient for fuzzy quantities;

- to substantiate the concept of characteristic functions of fuzzy quantities;

- to prove the limit theorem for the sum of fuzzy quantities.
4. Methods for solving problems of extending

the conceptual and analytical framework of the fuzzy set theory

4. 1. Development of a scheme for calculating the distribution density of fuzzy quantities

Let $\mu(x)$ be the membership function of the fuzzy quantity $x$. This function is not normalized, that is:

$$
\int_{-\infty}^{\infty} \mu(x) \mathrm{d} x \neq 1
$$

To solve many practical problems, it is useful to introduce a normalized membership function of the fuzzy quantity $x$ by the formula:

$$
\phi(x)=\frac{\mu(x)}{\int_{-\infty}^{\infty} \mu(x) \mathrm{d} x} .
$$

The resulting function can be interpreted as the density of the uncertainty measure and is naturally called the distribution density of the fuzzy quantity values. Using (1), we obtain the distribution function of the fuzzy quantity values in accordance with the relation:

$$
F(x)=\int_{-\infty}^{x} \phi(u) \mathrm{d} u=\int_{-\infty}^{x} \frac{\mu(u) \mathrm{d} u}{\int_{-\infty}^{\infty} \mu(u) \mathrm{d} u}=\frac{\int_{-\infty}^{x} \mu(u) \mathrm{d} u}{\int_{-\infty}^{\infty} \mu(u) \mathrm{d} u} .
$$

Function (2) has a clear meaning. For a given value of $x$, the value of $F(x)$ determines the chance, degree of confidence that the fuzzy quantity $u$ with the membership function $\mu(u)$ will take a value less than $x$, that is:

$$
F(x)=P(u<x)
$$

The function $F(x)$ has important properties:

$$
\begin{aligned}
& \text { a) } F(-\infty)=\int_{-\infty}^{-\infty} \phi(u) \mathrm{d} u=0, \\
& F(+\infty)=\int_{-\infty}^{\infty} \phi(u) \mathrm{d} u=\frac{\int_{-\infty}^{\infty} \mu(u) \mathrm{d} u}{\int_{-\infty}^{\infty} \mu(u) \mathrm{d} u}=1 ;
\end{aligned}
$$

b) if $a<b$, then

$$
\begin{aligned}
& F(b)-F(a)=\int_{-\infty}^{b} \phi(u) \mathrm{d} u-\int_{-\infty}^{a} \phi(u) \mathrm{d} u= \\
& =\int_{a}^{b} \phi(u) \mathrm{d} u=\frac{\int_{a}^{b} \mu(u) \mathrm{d} u}{\int_{-\infty}^{\infty} \mu(u) \mathrm{d} u}>0,
\end{aligned}
$$

that is, $F(x)$ is a non-decreasing function.

Relation (3) allows calculating the chance of falling of the fuzzy quantity $u$ with the membership function $\mu(u)$ into the range $[a, b]$. 
Note that the given properties of the distribution function $F(x)$ of the values of the fuzzy quantity $x$ coincide with the corresponding properties of the distribution functions of random variables, which allows using many important results of the probability theory in the fuzzy set theory.

Expected value of fuzzy quantity. The concept of expected value of fuzzy quantity as a natural analogue of mathematical expectation for the random variable was introduced in [4]. We introduce an alternative relation using the distribution density of the values of the fuzzy quantity $x$, which, by analogy with the corresponding formula of the probability theory, has the following form:

$$
M[x]=\int_{-\infty}^{\infty} x \phi(x) \mathrm{d} x=\frac{\int_{-\infty}^{\infty} x \mu(x) \mathrm{d} x}{\int_{-\infty}^{\infty} \mu(x) \mathrm{d} x} .
$$

It is clear that the expected value of the fuzzy quantity determined by (4) has all the basic properties of the mathematical expectation of a random variable:

1. If $x$ is a fuzzy quantity with the expected value $M[x]$, the expected value of the fuzzy quantity $a x$ is $M[a x]=a M[a x]$.

2. If $x$ and $y$ are fuzzy quantities with the expected values $M[x]$ and $M[y]$, respectively, the expected value of the sum of these fuzzy quantities is equal to the sum of their expected values, that is:

$$
M[x+y]=M[x]+M[y] .
$$

Example. Let the fuzzy quantity $x$ have the membership function:

$$
\mu(x)= \begin{cases}1, & x \in[a, b], \\ 0, & x \notin[a, b] .\end{cases}
$$

We find the expected value of the fuzzy quantity $x$. We have:

$$
\phi(x)=\frac{\mu(x)}{\int_{-\infty}^{\infty} \mu(x) \mathrm{d} x}=\frac{\mu(x)}{\int_{a}^{b} \mathrm{~d} x}= \begin{cases}\frac{1}{b-a}, & x \in[a, b], \\ 0, & x \notin[a, b] .\end{cases}
$$

Example. Let the fuzzy quantity $x$ have the triangular membership function:

$$
\mu(x)= \begin{cases}0, & x<a, \\ \frac{x-a}{c-a}, & x \in[a, c], \\ \frac{b-x}{b-c}, & x \notin[c, b], \\ 0, & x>b .\end{cases}
$$

We find the expected value of the fuzzy quantity $x$. We have:

$$
\phi(x)=\frac{\mu(x)}{\int_{-\infty}^{\infty} \mu(x) \mathrm{d} x}=\frac{2}{b-a} \mu(x) .
$$

Then

$$
\begin{aligned}
& M[x]=\int_{a}^{b} x \phi(x) \mathrm{d} x=\frac{2}{b-a}\left[\begin{array}{l}
\frac{1}{c-a} \int_{a}^{c} x(x-a) \mathrm{d} x+ \\
+\frac{1}{b-c} \int_{c}^{b} x(b-x) \mathrm{d} x
\end{array}\right]= \\
& =\frac{2}{b-a}\left[\begin{array}{c}
\frac{1}{c-a} \int_{a}^{c} x^{2} \mathrm{~d} x-\frac{a}{c-a} \int_{a}^{c} x \mathrm{~d} x+ \\
+\frac{b}{b-c} \int_{c}^{b} x \mathrm{~d} x-\frac{1}{b-c} \int_{c}^{b} x^{2} \mathrm{~d} x
\end{array}\right]= \\
& =\frac{2}{b-a}\left[\begin{array}{c}
\left.\frac{1}{c-a}\left(\frac{c^{3}}{3}-\frac{a^{3}}{3}\right)-\frac{a}{c-a}\left(\frac{c^{2}}{2}-\frac{a^{2}}{2}\right)+\right] \\
+\frac{b}{b-c}\left(\frac{b^{2}}{2}-\frac{c^{2}}{2}\right)-\frac{1}{b-c}\left(\frac{b^{3}}{3}-\frac{c^{3}}{3}\right)
\end{array}\right]= \\
& =\frac{2}{b-a}\left[\begin{array}{l}
\frac{1}{3}\left(c^{2}+a c+a^{2}\right)-\frac{a}{2}(c+a)+ \\
+\frac{b}{2}(b+c)-\frac{1}{3}\left(b^{2}+b c+c^{2}\right)
\end{array}\right]= \\
& =\frac{2}{b-a}\left[\begin{array}{c}
\frac{c^{3}}{3}+\frac{a c}{3}+\frac{a^{2}}{3}-\frac{a c}{2}-\frac{a^{2}}{2}+ \\
+\frac{b^{2}}{2}+\frac{b c}{2}-\frac{b^{2}}{3}-\frac{b c}{3}-\frac{c^{2}}{3}
\end{array}\right]= \\
& =\frac{2}{b-a}\left(-\frac{a c}{6}-\frac{a^{2}}{6}+\frac{b^{2}}{6}+\frac{b c}{6}\right)= \\
& =\frac{1}{3(b-a)}\left[b^{2}-a^{2}+c(b-a)\right]= \\
& =\frac{b-a}{3(b-a)}(a+b+c)=\frac{(a+b+c)}{3} \text {. }
\end{aligned}
$$

$$
\begin{aligned}
& M[g(x)]=\int_{-\infty}^{\infty} g(x) \mathrm{d} F(x)=\int_{-\infty}^{\infty} g(x) \phi(x) \mathrm{d} x= \\
& =\frac{1}{\int_{-\infty}^{\infty} \mu(x) \mathrm{d} x} \int_{-\infty}^{\infty} g(x) \mu(x) \mathrm{d} x .
\end{aligned}
$$

Relation (6) allows calculating moments of fuzzy quantities. By analogy with the terms of the probability theory, we call the quantity $M\left[x^{m}\right]$ the initial $k$-th moment of the fuzzy quantity $x$. By the same analogy, we call the quantity $a_{m}=M\left[(x-M[x])^{m}\right]$ the $m$-th central moment of the fuzzy quantity $x$. Let $\phi(x)$ be the distribution function of the values of the fuzzy quantity $x-M[x]$ and $g(x-M[x])=\left[(x-M[x])^{2}\right.$. Then, according to (6), we have:

$$
\begin{aligned}
& M\left[(x-M[x])^{2}\right]=\int_{-\infty}^{\infty}(x-M[x])^{2} \phi(x) \mathrm{d} x= \\
& =\int_{-\infty}^{\infty} x^{2} \phi(x) \mathrm{d} x-2 M[x] \int_{-\infty}^{\infty} x \phi(x) \mathrm{d} x+ \\
& +M^{2}[x] \int_{-\infty}^{\infty} \phi(x) \mathrm{d} x=\int_{-\infty}^{\infty} x^{2} \phi(x) \mathrm{d} x-M^{2}[x]
\end{aligned}
$$


Example. Let the fuzzy quantity $x$ have the membership function:

$$
\mu(x)= \begin{cases}1, & x \in[a, b], \\ 0, & x \notin[a, b] .\end{cases}
$$

We find the initial and second central moments of the fuzzy quantity $x$. Above is the formula for the distribution density of the values of the fuzzy quantity $x$, which has the following form:

$$
\phi(x)= \begin{cases}\frac{1}{b-a}, & x \in[a, b], \\ 0, & x \notin[a, b] .\end{cases}
$$

Then

$$
\begin{aligned}
& M\left[x^{2}\right]=\int_{-\infty}^{\infty} x^{2} \phi(x) \mathrm{d} x=\frac{1}{b-a} \int_{a}^{b} x^{2} \mathrm{~d} x= \\
& =\frac{1}{3} \frac{b^{3}-a^{3}}{b-a}=\frac{1}{3}\left(b^{2}+a b+a^{2}\right) .
\end{aligned}
$$

Further, since $M[x]=(a-b) / 2$, then

$$
\begin{aligned}
& M\left[(x-M[x])^{2}\right]=\int_{-\infty}^{\infty} x^{2} \phi(x) \mathrm{d} x-M^{2}[x]= \\
& =\frac{1}{3}\left(b^{2}+a b+a^{2}\right)-\frac{(a+b)^{2}}{4}=\frac{(b-a)^{2}}{12} .
\end{aligned}
$$

Example. Let the fuzzy quantity $x$ have the Gaussian membership function:

$$
\mu(x)=\exp \left\{-\frac{(x-m)^{2}}{2 \sigma^{2}}\right\} .
$$

We find the second central moment of the fuzzy quantity $x$. We have:

$$
\begin{aligned}
& M\left[(x-M[x])^{2}\right]= \\
& =\int_{-\infty}^{\infty}(x-m)^{2} \frac{1}{\sqrt{2 \pi} \sigma} \exp \left\{-\frac{(x-m)^{2}}{2 \sigma^{2}}\right\} \mathrm{d} x .
\end{aligned}
$$

As a result of substitution $u=(x-m) / \sigma$, we have:

$$
M\left[(x-M[x])^{2}\right]=\frac{\sigma^{2}}{\sqrt{2 \pi}} \int_{-\infty}^{\infty} u^{2} e^{-\frac{u^{2}}{2}} \mathrm{~d} u .
$$

Integrating by parts, we get $M\left[\left(x-M\left[x^{2}\right]\right)^{2}\right]=\sigma^{2}$.

Expressions for the moments of fuzzy quantities obtained in the examples naturally coincide with the corresponding expressions for random analogs of these quantities.

\section{2. Independent and dependent fuzzy quantities}

Let the fuzzy quantities $x_{1}, x_{2}, \ldots, x_{n}$ with the membership functions $\mu_{k}\left(x_{k}\right), k=1,2, \ldots, n$, belong to the corresponding sets $A_{1}, A_{2}, \ldots, A_{n}$, that is, $x_{k} \in A_{k}, k=1,2, \ldots, n$. We introduce the multidimensional quantity $X$ with coordinates $x_{1}, x_{2}, \ldots, x_{n}$, a multidimensional set $A=A_{1} \times A_{2} \times \ldots \times A_{n}$, and a scalar membership function $\mu\left(x_{1}, x_{2}, \ldots, x_{n}\right)$ of the vector argument $X$. The fuzzy quantities $x_{1}, x_{2}, \ldots, x_{n}$ can be independent or de- pendent. If they are independent, then to study them it is enough to know exactly the set of homogeneous membership functions $\mu_{1}\left(x_{1}\right), \mu_{2}\left(x_{2}\right), \ldots, \mu_{n}\left(x_{n}\right)$, each of which, for example, $\mu_{k}\left(x_{k}\right)$ specifies the degree of membership $x_{k}$ in the corresponding set $A_{k}$. In this case, the multidimensional function $\mu\left(x_{1}, x_{2}, \ldots, x_{n}\right)$ can be expressed through a set of one-dimensional functions as follows $\mu\left(x_{1}, x_{2}, \ldots, x_{n}\right)=\prod_{k=1}^{n} \mu_{k}\left(x_{k}\right)$.

Expected value of the product of independent fuzzy quantities. If the fuzzy quantities $x_{1}, x_{2}, \ldots, x_{n}$ are independent, then the expected value of the product of the fuzzy quantities is equal to the product of their expected values, that is:

$$
M\left[x_{1}, x_{2}, \ldots, x_{n}\right]=\prod_{k=1}^{n} M_{k}\left[x_{k}\right]
$$

Dependent fuzzy quantities. Calculation of the measure of connection of dependent quantities. Let's define the concept of a conditional membership function. Let the membership function $\mu\left(x_{1}, x_{2}, \ldots, x_{n}\right)$ be given for the multidimensional fuzzy quantity $\left(X_{1}+X_{2}+\ldots+X_{m}\right)$. Let us introduce the conditional function of membership of the component $X_{i}$ in the subset $A_{i}$, calculated under the condition that the other components of the set $\left(X_{1}, X_{2}, \ldots, X_{m}\right)$ took a certain value. This membership function is written as $\mu\left(x_{i}, x_{1}, x_{2}, \ldots, x_{i-1}, x_{i+1}, x_{m}\right)=$ $=\mu\left(x_{i}\right), i=1,2, \ldots, m$. In this case, the value of specific numerical values of any components of the fuzzy number $X$ does not affect the distribution of possible values for other components of this number.

If fuzzy variables are dependent, then knowledge of marginal membership functions is not enough to correctly describe them.

Consider the method for calculating the measure of connection of dependent fuzzy quantities. The idea of the method is extremely simple and especially clear in the two-dimensional case. The procedure is based on studying configurations of sections of the membership function of the multidimensional fuzzy number at several levels. It is clear that these configurations will be close to circular if the components of the fuzzy number are independent. And, on the contrary, they will be the more elliptical, the stronger their connection. This connection can be estimated quantitatively by calculating the static characteristics of a random variable evenly distributed within each of the sections. Let us take a closer look at this technology.

Let the joint membership function $\mu(x, y)$ of the fuzzy quantities $X$ and $Y$ be given. For this membership function, we find a set of $\alpha$ - level sections by solving the equations $\mu(x, y)=a_{1}, l=1,2, \ldots, L$. The solution of this equation for a specific value of $\alpha_{l}$ defines the two-dimensional domain $D_{l}$. We now assume that the variables $(x, y) \in D_{l}$ are a pair of random variables evenly distributed over the domain $D_{l}$. The corresponding two-dimensional distribution density is:

$$
f_{l}(x, y)=\left\{\begin{array}{ll}
1 / s_{l}, & (x, y) \in D_{l}, \\
0, & (x, y) \notin D_{l},
\end{array} \quad s_{l}=\iint_{D_{l}} \mathrm{~d} x \mathrm{~d} y .\right.
$$

Now, for the fixed value of $x$, we find the interval $\left[d_{1 l}(x), d_{2 l}(x)\right]$ of possible values of $y$ for which $(x, y) \in D_{l}$. Then the conditional distribution density of the variable $X$ is:

$$
f_{1 l}(x)=\int_{-\infty}^{\infty} f(x, y) \mathrm{d} y=\int_{d_{1 l}(x)}^{d_{2 l}(x)} \frac{1}{s_{l}} \mathrm{~d} y=\frac{d_{2 l}(x)-d_{1 l}(x)}{s_{l}} .
$$


Similarly, for the fixed value of $y$, we find the interval [ $\left.d_{1 l}(y), d_{2 l}(y)\right]$ of possible values of $x$ for which $(x, y) \in D_{l}$. In this case, the conditional distribution density of the variable $Y$ is:

$$
f_{2 l}(Y)=\int_{-\infty}^{\infty} f(X, Y) \mathrm{d} x=\int_{d_{1 l}(y)}^{d_{2 l}(y)} \frac{1}{s_{l}} \mathrm{~d} x=\frac{d_{2 l}(y)-d_{1 l}(y)}{s_{l}} .
$$

Let us determine the values of mathematical expectations and variances of $X$ and $Y$ :

$$
\begin{aligned}
& M_{l}[X]=\int_{-\infty}^{\infty} x f_{1 l}(x) \mathrm{d} x=\frac{1}{s_{l}} \int_{X_{\min }^{l}}^{X_{\max }^{l}} x\left(d_{2 l}(x)-d_{1 l}(x)\right) \mathrm{d} x ; \\
& M_{l}[Y]=\int_{-\infty}^{\infty} y f_{2 l}(y) \mathrm{d} y=\frac{1}{s_{l}} \int_{Y_{\min }^{l}}^{Y_{\max }} y\left(d_{2 l}(y)-d_{1 l}(y)\right) \mathrm{d} y ; \\
& M_{l}\left[X^{2}\right]=\int_{-\infty}^{\infty} x^{2} f_{1 l}(x) \mathrm{d} x=\frac{1}{s_{l}} \int_{X_{\min }^{l}}^{X_{\max }^{l}} x^{2}\left(d_{2 l}(x)-d_{1 l}(x)\right) \mathrm{d} x ; \\
& M_{l}\left[Y^{2}\right]=\int_{-\infty}^{\infty} y^{2} f_{1 l}(y) \mathrm{d} y=\frac{1}{s_{l}} \int_{Y_{\min }^{l}}^{Y_{\max }^{l}} y^{2}\left(d_{2 l}(y)-d_{1 l}(y)\right) \mathrm{d} y \\
& D_{l}[X]=M_{l}\left[X^{2}\right]-\left(M_{l}[X]\right)^{2} ; \\
& D_{l}[Y]=M_{l}\left[Y^{2}\right]-\left(M_{l}[Y]\right)^{2} .
\end{aligned}
$$

Finally, let us calculate the covariance for the random variables $X$ and $Y$, corresponding to the $\alpha_{l}-$ level section of the membership function $\mu(x, y)$.

$$
\begin{aligned}
& M_{l}[X Y]=\int_{-\infty}^{\infty} \int_{-\infty}^{\infty} x y f(x y) \mathrm{d} x \mathrm{~d} y=\frac{1}{s_{l}} \int_{X_{\min }^{l}}^{X_{\max }^{l}} x \mathrm{~d} x \int_{d_{1 l}(x)}^{d_{2 l}(x)} y \mathrm{~d} y= \\
& =\frac{1}{2 s_{l}} \int_{X_{\min }^{l}}^{X_{\max }^{l}} x\left(d_{2 l}^{2}(x)-d_{1 l}^{2}(x)\right) \mathrm{d} x .
\end{aligned}
$$

Then $K_{l}[X Y]=M_{l}[X Y]-M_{l}[X] M_{l}[Y]$.

We repeat the described procedure for all $\alpha_{l}, l=1,2, \ldots, L$, and obtain the corresponding set of covariance values $K_{1}[X Y]$, $K_{2}[X Y], \ldots, K_{L}[X Y]$. Now, as a measure of connection $\operatorname{con}(X, Y)$ for two fuzzy quantities $X$ and $Y$, we take the weighed linear combination of the calculated values of $\alpha_{l}-$ level covariances:

$$
\operatorname{con}(X, Y)=\sum_{l=1}^{L} \frac{\alpha_{l}}{\sum_{l=1}^{L} \alpha_{l}} K_{l}[X, Y] .
$$

The above values of the variances of the random variables $X$ and $Y$ for the $\alpha$-sections of the membership function $\mu(x, y)$ allow us to find the value of the relative connection coeffi$\operatorname{cient}-\operatorname{ccon}(X, Y)$ for $X$ and $Y$ :

$$
\operatorname{ccon}(X, Y)=\sum_{l=1}^{L} \frac{\alpha_{l}}{\sum_{l=1}^{L} \alpha_{l}} \frac{K_{l}[X, Y]}{\left(D_{l}[X] D_{l}[Y]\right)^{\frac{1}{2}}} .
$$

It is clear that in cases where the analytical description of the domain $D_{l}$ is complex, then to obtain the desired result, a simulation model can be used to obtain the desired result by forming random variables uniformly distributed in $D_{l}$.

\section{3. Characteristic function of a fuzzy quantity}

By analogy with the concept of the characteristic function of a random variable, the characteristic function of the fuzzy quantity $x$ is the function defined by the relation:

$$
\Psi(z)=M\left[e^{i z x}\right]=M[\cos z x+i \sin z x] .
$$

For the fuzzy quantity with the distribution density of values $\phi(x)$, the characteristic function according to (8) is calculated by the formula:

$$
\Psi(z)=\int_{-\infty}^{\infty} e^{i z x} \phi(x) \mathrm{d} x
$$

Example. For the fuzzy quantity $x$ with the membership function:

$$
\mu(x)= \begin{cases}1, & x \in[a, b], \\ 0, & x \notin[a, b] .\end{cases}
$$

as was shown, the distribution density of values is:

$$
\phi(x)= \begin{cases}1 /(b-a), & x \in[a, b], \\ 0, & x \notin[a, b] .\end{cases}
$$

Let us find the characteristic function of the fuzzy number $x$. We have:

$$
\Psi(z)=\frac{1}{b-a} \int_{-\infty}^{\infty} e^{i z x} \mathrm{~d} x=\frac{e^{i z b}-e^{i z a}}{(b-a) i z} .
$$

In particular, if the fuzzy quantity carrier is the interval $[-a, a]$, then:

$$
\begin{aligned}
& \Psi(z)=\frac{1}{2 a i z}\left(e^{i z a}-e^{-i z a}\right)= \\
& =\frac{1}{2 a i z}(\cos z a+i \sin z a-\cos z a+i \sin z a)=\frac{\sin z a}{2 a} .
\end{aligned}
$$

Example. Let the fuzzy quantity $x$ have the Gaussian membership function:

$$
\mu(x)=\exp \left\{-\frac{x^{2}}{2}\right\} .
$$

Let us find the characteristic function of this fuzzy quantity. The distribution density of the values of this quantity is:

$$
\phi(x)=\frac{1}{\sqrt{2 \pi}} \exp \left\{-\frac{x^{2}}{2}\right\} .
$$

Then

$$
\Psi(z)=\frac{1}{\sqrt{2 \pi}} \int_{-\infty}^{\infty} e^{i z x} e^{-\frac{x^{2}}{2}} \mathrm{~d} x=\frac{1}{\sqrt{2 \pi}} \int_{-\infty}^{\infty} e^{i z x-\frac{x^{2}}{2}} \mathrm{~d} x .
$$

Further

$$
\frac{d \Psi(z)}{d z}=\frac{1}{\sqrt{2 \pi}} \int_{-\infty}^{\infty} i x e^{i z x-\frac{x^{2}}{2}} \mathrm{~d} x .
$$


Integrating the resulting expression by parts, we get:

$$
\begin{aligned}
& \frac{d \Psi(z)}{d z}=\frac{i}{\sqrt{2 \pi}} \int_{-\infty}^{\infty} e^{i z x}\left(-x e^{-\frac{x^{2}}{2}}\right) \mathrm{d} x= \\
& =-\frac{i}{\sqrt{2 \pi}}\left[\left.e^{i z x} e^{-\frac{x^{2}}{2}}\right|_{-\infty} ^{\infty}-i z \int_{-\infty}^{\infty} e^{i z x-\frac{x^{2}}{2}} \mathrm{~d} x\right]= \\
& =-\frac{z}{\sqrt{2 \pi}} \int_{-\infty}^{\infty} e^{i z x-\frac{x^{2}}{2}} \mathrm{~d} x=-z \Psi(z) .
\end{aligned}
$$

Thus, $\Psi(z)$ satisfies the differential equation $\Psi^{\prime}(z)=-z \Psi(z)$, solving which we obtain:

$$
\Psi(z)=C e^{-\frac{z^{2}}{2}} \text {. }
$$

Since

$$
\Psi(0)=\frac{1}{\sqrt{2 \pi}} \int_{-\infty}^{\infty} e^{-\frac{x^{2}}{2}} \mathrm{~d} x=1,
$$

then $C=1$ and $\Psi(z)=e^{-\frac{z^{2}}{2}}$. Let further:

$$
\phi(x)=\frac{1}{\sqrt{2 \pi} \sigma} \exp \left\{-\frac{(x-m)^{2}}{2 \sigma^{2}}\right\} .
$$

Let us introduce $\eta=(x-m) / \sigma$, whence $x=\sigma \eta+m$. Then

$$
\phi(\eta)=\frac{1}{\sqrt{2 \pi}} e^{-\frac{\eta^{2}}{2}}
$$

Moreover, using (8), we obtain:

$$
\begin{aligned}
& \Psi_{\eta}(z)=M\left[e^{i z x}\right]=M\left[e^{i z m} e^{i z \sigma \eta}\right]= \\
& =e^{i z m} M\left[e^{i z(\sigma \eta)}\right]=e^{i z m} e^{-\frac{(\sigma z)^{2}}{2}}=e^{i z m-\frac{(\sigma z)^{2}}{2}} .
\end{aligned}
$$

Note now the important multiplicative property of characteristic functions of fuzzy quantities. Let $x_{1}$ and $x_{2}$ be independent fuzzy quantities and $\Psi_{1}(z)$ and $\Psi_{2}(z)$ - their characteristic functions. Let us find the characteristic function for the sum $x_{1}+x_{2}$. We have:

$$
\begin{aligned}
& \Psi\left(x_{1}+x_{2}\right)=M\left[e^{i z\left(x_{1}+x_{2}\right)}\right]=M\left[e^{i z x_{1}} e^{i z x_{2}}\right]= \\
& =M\left[e^{i z x_{1}}\right] M\left[e^{i z x_{2}}\right]=\Psi_{1}(z) \Psi_{2}(z) .
\end{aligned}
$$

It follows that if $x_{1}, x_{2}, \ldots, x_{n}$ are independent fuzzy quantities, then the characteristic function of the sum $x_{1}+x_{2}+\ldots+x_{n}$ of these quantities is equal to the product of the characteristic functions of the terms.

Example. Let $x_{1}, x_{2}, \ldots, x_{n}$ be independent fuzzy quantities with Gaussian distribution densities of their values:

$$
\begin{aligned}
& \phi_{1}\left(x_{1}\right)=\frac{1}{\sqrt{2 \pi} \sigma_{1}} \exp \left\{-\frac{\left(x_{1}-m_{1}\right)^{2}}{2 \sigma_{1}^{2}}\right\}, \ldots, \\
& \phi_{n}\left(x_{n}\right)=\frac{1}{\sqrt{2 \pi} \sigma_{n}} \exp \left\{-\frac{\left(x_{n}-m_{n}\right)^{2}}{2 \sigma_{n}^{2}}\right\} .
\end{aligned}
$$

Using the properties of the characteristic functions, we find the distribution density of the values of the sum $x_{1}, x_{2}, \ldots, x_{n}$.
The characteristic functions for each of the terms are determined (11):

$$
\Psi_{k}(z)=e^{i z m_{k} \frac{\sigma_{k}^{2} z^{2}}{2}}, \quad k=1,2, \ldots, n .
$$

Then the characteristic function of the sum $x_{1}, x_{2}, \ldots, x_{n}$ equal to the product of the characteristic functions of the terms is as follows:

$$
\begin{aligned}
& \prod_{k=1}^{n} e^{i z m_{k}-\frac{\sigma_{k}^{2} z^{2}}{2}}= \\
& =e^{\sum_{k=1}^{n}\left(i z m_{k}-\frac{\sigma_{k}^{2} z^{2}}{2}\right)}=e^{i z\left(\sum_{k=1}^{n} m_{k}\right)-\frac{z^{2}}{2} \sum_{k=1}^{n} \sigma_{k}^{2}}=e^{i z m_{\Sigma}-\frac{z^{2}}{2} \sigma_{\Sigma}^{2}} .
\end{aligned}
$$

The resulting function according to (13) is the characteristic function of the fuzzy quantity with the distribution density of values:

$$
\phi(u)=\frac{1}{\sqrt{2 \pi} \sigma_{\Sigma}} \exp \left\{-\frac{\left(u-m_{\Sigma}\right)^{2}}{2 \sigma_{\Sigma}^{2}}\right\} .
$$

Thus, if fuzzy quantities have a Gaussian distribution density of values, then the distribution density of their sum is also Gaussian.

\section{4. Limit theorem for the sum of independent fuzzy} quantities

Let us show that the sum of a large number of independent fuzzy quantities has an asymptotically Gaussian distribution density (an analogue of the central limit theorem of the probability theory). Let $n$ independent fuzzy quantities $\xi_{k}, k=1,2, \ldots n$ be summed up, with respect to each of which the expected value $m_{k}$ and the second central moment $\sigma_{k}^{2}$ are known. The characteristic function of the $k$-th fuzzy quantity is:

$$
\Psi_{k}\left(\xi_{k}\right)=M\left[e^{i z \xi_{k}}\right]
$$

We introduce centered fuzzy quantities $\zeta_{k}=\xi_{k}-m_{k}$, where $k=1,2, \ldots, n$. Now using decomposition:

$$
e^{i z x}=\sum_{s=0}^{m} \frac{(i x)^{s}}{s !} z^{s}+\frac{(\theta x)^{m+1}}{(m+1) !} z^{m+1},|\theta|<1,
$$

we write the characteristic function of the fuzzy variable $\zeta_{k}$ :

$$
\begin{aligned}
& \Psi\left(\zeta_{k}\right)=M\left[e^{i z \zeta_{k}}\right]=M\left[\sum_{s=0}^{m} \frac{\left(i \zeta_{k}\right)^{s}}{s !} z^{s}+\frac{\left(\theta \zeta_{k}\right)^{m+1}}{(m+1) !} z^{m+1}\right]= \\
& =\sum_{s=0}^{m} \frac{i^{s}}{s !} M_{s}\left(\zeta_{k}\right) z^{s}+\frac{\theta^{m+1}}{(m+1) !} z^{m+1} M\left[\zeta_{k}^{m+1}\right] .
\end{aligned}
$$

Let the so-called Lyapunov condition be satisfied for the set of the summed quantities:

$$
\lim _{n \rightarrow \infty} \sum_{k=1}^{n} M\left[\zeta_{k}^{3}\right]=0 .
$$


In this case, (14) takes the form:

$$
\begin{aligned}
& \Psi\left(\zeta_{k}\right)=1+i M\left[\zeta_{k}\right] z+\frac{i^{2}}{2} M\left[\zeta_{k}^{2}\right] z^{2}+\frac{R}{(m+1) !} z^{m+1}= \\
& =1-\frac{1}{2 n} \sigma_{k}^{2} z^{2}+\frac{R}{(m+1) !} z^{m+1},
\end{aligned}
$$

where the variable $R$ is bounded from above, $|R| \leq M\left[\zeta_{k}^{m+1}\right]$.

Then the generating function for the sum of centered fuzzy quantities $\zeta_{k}$ is determined by the relation:

$$
\Psi\left(\sum_{k=1}^{n} \zeta_{k}\right)=\prod_{k=1}^{n} \Psi\left(\zeta_{k}\right)=\prod_{k=1}^{n}\left(1-\frac{1}{2 n} \sigma_{k}^{2} z^{2}+\frac{R}{(m+1) !} z^{m+1}\right) .
$$

Taking the logarithm of (15), we obtain:

$$
\begin{aligned}
& \ln \Psi\left(\sum_{k=1}^{n} \zeta_{k}\right)=\sum_{k=1}^{n} \ln \left(1-\frac{1}{2 n} \sigma_{k}^{2} z^{2}+\frac{R}{(m+1) !} z^{m+1}\right) \approx \\
& \approx \sum_{k=1}^{m}\left(-\frac{1}{2 n} \sigma_{k}^{2} z^{2}\right)=-\frac{1}{\Sigma} \sigma_{\Sigma}^{2} z^{2} \\
& \sigma_{\Sigma}^{2}=\sum_{k=1}^{n} \sigma_{k}^{2} .
\end{aligned}
$$

Hence:

$$
\Psi\left(\sum_{k=1}^{n} \zeta_{k}\right)=e^{-\frac{z^{2}}{2} \sigma_{\Sigma}^{2}} .
$$

It follows from (16) according to (13) that the sum $\sum_{k=1}^{n} \zeta_{k}$ has an asymptotically normal distribution with the parameters $\left(0, \sigma_{\Sigma}^{2}\right)$. On the other hand, since $\sum_{k=1}^{n} \zeta_{k}=\sum_{k=1}^{n} \xi_{k}-\sum_{k=1}^{n} m_{k}$, then $\sum_{k=1}^{n} \xi_{k}+\sum_{k=1}^{n} m_{k}$, whence:

$$
\begin{aligned}
& \Psi\left(\sum_{k=1}^{n} \xi_{k}\right)=\Psi\left(\sum_{k=1}^{n} \zeta_{k}\right) \Psi\left(\sum_{k=1}^{n} m_{k}\right)= \\
& =e^{-\frac{z^{2}}{2} \sigma_{\Sigma}} M\left[e^{i z m_{\Sigma}}\right]=e^{i z m_{\Sigma}-\frac{1}{2} z^{2} \sigma_{\Sigma}} \\
& m_{\Sigma}=\sum_{k=1}^{n} m_{k}
\end{aligned}
$$

which, taking into account (13), corresponds to normal distribution with the parameters $N\left(m_{\Sigma}, \sigma_{\Sigma}^{2}\right)$.

\section{Discussion of the results of extension of the conceptual and analytical framework of the fuzzy set theory (FST)}

The issues discussed in the work are a very small part of many FST problems that need to be worked out. The list of those chosen for consideration is, of course, subjective, but dictated by the urgent needs of practice.

For continuous fuzzy quantities, the concept of the distribution density of the values of these quantities, which is a natural analogue of the corresponding concept in the probability theory, is introduced. The absolute importance of this concept is due to the fact that it defines a probabilistic measure for fuzzy quantities. This measure is absolutely necessary when solving many problems of assessing the probability of specific events in the real world. In addition, knowledge of the distribution density of fuzzy quantities provides a calculation of the numerical characteristics of the values of these quantities, giving a concise, but rather an informative display of the essential features of mathematical models of the processes and objects under study. The technology introduced below for calculating the moments for functions of fuzzy quantities significantly expands the possibilities of using fuzzy mathematics to solve a variety of practical problems, the parameters of which are not clearly defined. These include, in particular, the following problems: linear and nonlinear algebraic equations and their systems, differential and integral equations, problems of unconditional and conditional optimization; decision making under fuzzy conditions; formation of mathematical models of systems functioning in a fuzzy environment, etc.

The concepts of independence and dependence for fuzzy quantities are considered. For the case of dependent fuzzy quantities, the technology for calculating the coefficient, the numerical value of which lies in the interval $[0 ; 1]$, determines the measure of connection between them, is proposed.

The concept is introduced and the method is proposed for calculating the characteristic function for fuzzy quantities. A characteristic function is one way to define a distribution. It is a very useful and convenient tool for analyzing distribution densities. Using characteristic functions, the problem of finding the result of the composition of distribution laws and many other problems are solved.

The limit behavior of the value of the sum of a large number of weakly connected fuzzy quantities is considered. The important property of the distribution of this value is determined - this distribution is asymptotically normal. This most important property of the sum of a large number of weakly connected fuzzy numbers can be constructively used in solving a variety of problems of mathematical statistics. These include, in particular, problems of regression and cluster analysis [15, 20], multivariate logistics [21, 22].

Note the following important circumstance. To solve problems using the introduced formalisms, a unified approach can be used, implemented by the following fourstage procedure. Let, for example, some specific problem be reduced to finding a set of fuzzy variables $X=\left(x_{1}, x_{2}, \ldots, x_{n}\right)$, given by their membership functions $\mu\left(x_{1}\right), \mu\left(x_{2}\right), \ldots, \mu\left(x_{n}\right)$, which maximizes the objective function $F(x)$.

At the first stage, the usual problem of maximizing $F(\bar{x})$, is extended, $\bar{X}=\left(\bar{x}_{1}, \bar{x}_{2}, \ldots, \bar{x}_{n}\right)-$ a set of modal values of variables. Let $X^{(0)}=\left(x_{1}^{(0)}, x_{2}^{(0)}, \ldots, x_{n}^{(0)}\right)$ be the solution to this problem.

At the second stage, using the membership functions of the variables $x_{i}, i=1,2, \ldots, n$, their distribution density $\phi\left(x_{i}\right)$, $i=1,2, \ldots, n$ is formed.

At the third stage, using $\mu\left(x_{1}\right), \mu\left(x_{2}\right) \ldots, \mu\left(x_{n}\right)$, the membership function of the objective function of the problem $\mu(F(x))$ and the variance of this fuzzy number $D[F(x)]$ are determined.

At the fourth stage, a compromise criterion for the quality of solving the problem:

$$
\eta(x)=\alpha_{1} D[F(x)]+\alpha_{2}\left(x-x^{(0)}\right)^{T}\left(x-x^{(0)}\right),
$$

is formed, which is minimized. 
The essence of this operation is clear: the level of fuzziness of the objective function value is minimized, as well as the degree of deviation of the solution from the modal one.

In conclusion, we note that the implementation of the computational capabilities of the proposed methods is the simplest and the most convenient if fuzzy operands are specified by membership functions of the $(L-R)$ type. These techniques are applicable in other cases, however, computational difficulties increase significantly.

A possible direction for further research is the extension of the results to the case when the initial data are fuzzy [23]. In this case, the approaches proposed in [24] can be useful.

\section{Conclusions}

1. The method for calculating the distribution density of a fuzzy quantity is developed, the concept is introduced and the method is determined for calculating its expected value.

2. The concepts of independence and dependence for fuzzy quantities are introduced and substantiated. The method for calculating the connection of dependent fuzzy quantities is proposed.

3. The concept of characteristic functions of fuzzy quantities is introduced. The formula for their calculation is proposed.

4. Using the characteristic functions, the central limit theorem for the sum of a large number of weakly connected fuzzy quantities is proved.

\section{References}

1. Zadeh, L. A. (1965). Fuzzy sets. Information and Control, 8 (3), 338-353. doi: https://doi.org/10.1016/s0019-9958(65)90241-x

2. Negoytse, K. (1981). Primenenie teorii sistem k problemam uravleniya. Moscow: MIR, 219.

3. Orlovskiy, S. A. (1981). Problemy prinyatiya resheniy pri nechetkoy informatsii. Moscow: Nauka, 264.

4. Dyubua, D., Prad, A. (1990). Teoriya vozmozhnostey. Prilozhenie k predstavleniyu znaniy v informatike. Moscow: Radio i svyaz', 286.

5. Kofman, A. (1982). Vvedenie v teoriyu nechetkih mnozhestv. Moscow: Radio i svyaz', 486.

6. Lyu, B. (2005). Teoriya i praktika neopredelennogo programmirovaniya. Moscow: BINOM, 416.

7. Leonenkov, A. V. (2003). Nechetkoe modelirovanie v srede Matlab i fuzzyTech. Sankt-Peterburg: BHV - Peterburg, 736.

8. Raskin, L. G., Seraya, O. V. (2008). Nechetkaya matematika. Kharkiv: Parus, 352.

9. Ryzhov, A. P. (1998). Elementy teorii nechetkih mnozhestv i izmereniya nechetkosti. Moscow: Dialog MGU, 116.

10. Borisov, V. V., Fedulov, A. S., Zernov, M. M. (2014). Osnovy teorii nechetkih mnozhestv. Moscow: Goryachaya Liniya - Telekom, 88.

11. Ponomarev, A. S. (2005). Nechetkie mnozhestva v zadachah avtomatizirovannogo upravleniya i prinyatiya resheniy. Kharkiv: $\mathrm{NTU} \ll \mathrm{KhPI}, 232$.

12. Yahyaeva, G. (2012). Nechetkie mnozhestva i neyronnye seti. Moscow: Internet-Universitet Informatsionnyh tehnologiy; BINOM. Laboratoriya znaniy, 316.

13. Borisov, V. V., Kruglov, V. V., Fedulov, A. S. (2007). Nechetkie modeli i seti. Moscow: Goryachaya Liniya - Telekom, 284.

14. Zimmermann, H.-J. (2010). Fuzzy set theory. Wiley Interdisciplinary Reviews: Computational Statistics, 2 (3), $317-332$. doi: https://doi.org/10.1002/wics.82

15. Timm, H., Borgelt, C., Döring, C., Kruse, R. (2004). An extension to possibilistic fuzzy cluster analysis. Fuzzy Sets and Systems, 147 (1), 3-16. doi: https://doi.org/10.1016/j.fss.2003.11.009

16. Gottwald, S. (2006). Universes of Fuzzy Sets and Axiomatizations of Fuzzy Set Theory. Part I: Model-Based and Axiomatic Approaches. Studia Logica, 82 (2), 211-244. doi: https://doi.org/10.1007/s11225-006-7197-8

17. Kremer, N. Sh. (2004). Teoriya veroyatnostey i matematicheskaya statistika. Moscow: YUNITI - DANA, 573.

18. Chernova, N. I. (2007). Teoriya veroyatnostey. Novosibirsk, 160.

19. Natan, A. A., Gorbachev, O. G., Guz, S. A. (2007). Teoriya veroyatnostey. Moscow: MFTI, 253.

20. Raskin, L., Sira, O., Ivanchykhin, Y. (2017). Models and methods of regression analysis under conditions of fuzzy initial data. Eastern-European Journal of Enterprise Technologies, 4 (4 (88)), 12-19. doi: https://doi.org/10.15587/1729-4061.2017.107536

21. Raskin, L., Sira, O., Karpenko, V. (2017). Calculation of throughputs of intermediate centers in three-index transportation problems. Eastern-European Journal of Enterprise Technologies, 3 (4 (87)), 31-37. doi: https://doi.org/10.15587/1729-4061.2017.103950

22. Raskin, L., Sira, O., Karpenko, V. (2019). Transportation management in a distributed logistic consumption system under uncertainty conditions. EUREKA: Physics and Engineering, 4, 82-90. doi: https://doi.org/10.21303/2461-4262.2019.00936

23. Pawlak, Z. (1991). Rough Sets: Theoretical Aspects of Reasoning about Data. Springer. doi: https://doi.org/10.1007/978-94-011-3534-4

24. Raskin, L., Sira, O. (2016). Method of solving fuzzy problems of mathematical programming. Eastern-European Journal of Enterprise Technologies, 5 (4 (83)), 23-28. doi: https://doi.org/10.15587/1729-4061.2016.81292 